\title{
Clostridium difficile Infection: Considerations for Hospitalists
}

\author{
Kristin Hahn-Cover ${ }^{1,2}$ \\ ${ }^{1}$ Division of Hospital Medicine, Department of Medicine, University of Missouri Health Care, Columbia, MO. \\ ${ }^{2}$ Chief Quality Officer, University of Missouri Health Care, Columbia, MO. \\ Correspondence: Kristin Hahn-Cover (HahnCoverK@ health.missouri.edu) \\ Received November 30, 2016; accepted February 20, 2017
}

\section{Introduction}

As hospitalists, we often care for patients who either present with diarrhea or develop diarrhea during their hospital visit. We have learned to think of Clostridium difficile infection (CDI) early. In the United States, administrative data demonstrated significant increases in hospitalized patients with CDI beginning in 2001 (1). Discharge diagnosis of CDI more than doubled between 2000 and 2009. In 2011, Clostridium difficile was responsible for more than 450,000 infections and more than 29,000 deaths in the United States (2). Patient outcomes associated with hospital-acquired infection include increased mortality, length of stay and rate of discharge to long-term care (3). This infection is of particular interest in the healthcare setting, given the risk of environmental exposure and healthcare transmission of infection via the spore form of the organism.

\section{Case definition}

The case definition for Clostridium difficile infection is a patient with a) three or more unformed stools within 24 hours, and b) either a positive stool test or diagnosis of pseudomembranous colitis. Patients may be asymptomatic carriers, colonized by $C$. difficile instead of infected by the organism. In fact, the literature demonstrates " $50 \%$ or more of hospital patients colonized by C. difficile are symptomless carriers..." (1). Transmission is via the fecal-oral route, and $C$. difficile spores are present on the skin and in the environment of colonized and infected patients (3). The Centers for Disease Control and Prevention (CDC) define a hospital's CDI laboratoryidentified (LabID) Event Reporting to the National Healthcare Safety Network (NHSN). Definitions distinguish between community-onset infection, when the specimen was collected within 3 days of admission; and healthcare facility-onset, when the specimen was collected $>3$ days after admission (4). Estimated time from exposure to development of infection is 2-3 days (1). In the NHSN LabID CDI Event Reporting definition, a test is positive when either $C$. difficile toxin A and/or B is positive or toxin-producing $C$. difficile organism is detected, and the stool specimen tested is unformed. Any subsequent positive test result within two weeks in the same facility is considered a duplicate test (4).

\section{Diagnostic methods}

Commonly used diagnostic tests include enzyme immunoassay (EIA) tests for toxin A and/or B and polymerase chain reaction (PCR) for toxin B detection. The test in use in our organization, 
University of Missouri Health Care, is the Xpert C. difficile/Epi, a PCR-based test with sensitivity $98.7 \%$ and specificity $90.9 \%$, as reported in the package insert (5). We typically use diagnostic testing to determine a patient's treatment plan, and to consider isolation and environmental disinfection needs. A positive test does not distinguish between CDI and asymptomatic colonization, and positive predictive value is strongly influenced by the pre-test probability of infection (6). Patients who have achieved clinical cure of infection with treatment will continue to test positive for weeks, and testing stool for clearance is not appropriate or necessary (3).

\section{Prevention}

Our most effective efforts to prevent Clostridium difficile infection are hand hygiene and antimicrobial stewardship. While $C$. difficile spores remain infectious in the environment, patient exposure to $C$. difficile in the healthcare setting is primarily attributable to the practices of healthcare workers (HCW) (3). Because alcohol does not kill $C$. difficile spores, healthcare workers are advised to use soap and water for hand hygiene after providing care for a patient with CDI. While soap and water do not kill spores, this handwashing practice removes spores from HCW hands. HCW use of gloves is another essential element of hand hygiene in protecting patients from hospital-acquired CDI, and gowns complete the contact precautions for body substances and environmental contamination (7). In fact, these contact precautions are indicated when caring for hospitalized patients with diarrheal illness in advance of the return of positive test results. Other recommended hospital practices are using private rooms and dedicated patient care items for infected patients, and environmental disinfection with specific products and equipment. These practices are not recommended for patients who are asymptomatic carriers (3).

Antibiotics increase patient susceptibility to Clostridium difficile infection by disrupting gut flora, and antimicrobial stewardship programs to reduce antibiotic utilization and modify antibiotic use patterns are increasingly important. C. difficile infection risk is highest within the first 30 days after antibiotic exposure, but persists as long as 90 days. The particular antibiotic class risk for CDI varies by patient population, with published analyses differentiating between risk for hospital-acquired and community-acquired infection. In general, broader spectrum and Gram-negative or anaerobic activity increase risk. Clindamycin exposure is associated with increased risk (8). Third-generation cephalosporins present the most risk as a class, and fluoroquinolones present risk for a particular fluoroquinolone-resistant strain (9). When a patient at risk of CDI develops diarrhea, the first tactic in treatment is to discontinue non-CDI antibiotic therapy as soon as it is no longer needed for other suspected or proven bacterial infection.

\section{Treatment}

Antibiotic therapy specific to CDI should be started when the diagnosis is made, and empiric treatment specific to CDI may be appropriate before laboratory confirmation in severe or complicated illness. First-line therapy is metronidazole $500 \mathrm{mg}$ orally, three times daily, with a 10-14 day duration of therapy, when illness is not severe. In severe illness, vancomycin is the drug of choice, dosed at $125 \mathrm{mg}$ orally, four times daily, with a 10-14 duration of therapy. In severe, complicated CDI, the dose of vancomycin is increased to $500 \mathrm{mg}$ four times daily, intravenous metronidazole is added, and vancomycin retention enema is required if patients have 
ileus (1). Surgical consultation is appropriate, particularly when the white cell count and/or serum lactate is increasing, as colectomy may be necessary. Of note, patients with hospitalacquired infection are at highest risk for recurrence after clinical cure, with estimated first recurrence rate of $21 \%$ (2). The first recurrence of CDI is usually treated with the same regimen as for the initial episode, either with metronidazole or vancomycin, depending on severity. Subsequent recurrences should be treated with vancomycin (1). Other treatments with evidence of benefit are nitazoxanide, rifaximin and fidaxomicin, probiotics, and fecal bacteriotherapy or "fecal transplant" (10). Infectious Diseases and Gastroenterology consultations may be required for management of recurrences.

\section{Impact of CDI on hospital finances}

Rates of hospital-acquired infection factor in to the Centers for Medicare and Medicaid Services (CMS) reporting and payment programs. Reporting hospital-onset CDI to the NHSN is required for payment determination as part of the Inpatient Quality Reporting (IQR) program. In the Fiscal Year 2018 Hospital Value Based Purchasing program, 2\% of hospital Medicare payment is redistributed based on performance in four categories: clinical care, safety, experience of care/care coordination, and efficiency/cost reduction. CDI rates are contained within the safety category, as one of seven elements that in aggregate comprise $25 \%$ of the performance score determining value-based payment. In the Hospital Acquired Conditions Reduction Program, hospitals in the most poorly-performing quartile are penalized $1 \%$ of hospital Medicare payments. Eighty-five percent of the performance score is determined by hospital-acquired infections, and CDI is one of five in the category (11). Together, these programs provide incentives beyond excellent patient care to avoid hospital-onset Clostridium difficile infections, through reputational impact of public reporting and financial pressures from payment cuts.

\section{Take-home points}

- Clostridium difficile infection (CDI) is increasing in frequency, and is associated with mortality and longer length of stay

- Definition of CDI is diarrhea (3+ unformed stools within 24 hours) and positive stool test; patients with clinical cure and colonization may have positive stool test but do not have CDI

- Patients with non-severe CDI should be treated with metronidazole and patients with severe CDI should be treated with oral vancomycin. Either metronidazole or vancomycin may be used for recurrent illness, depending on severity; metronidazole should not be used beyond the first recurrence

- Patients with CDI in the hospital require contact isolation, with healthcare workers using gown, gloves, and hand hygiene with soap and water to minimize risk of transmission to others; isolation is not indicated for colonized patients

- Antimicrobial stewardship reduces patient risk of CDI

- Hospital-acquired CDI rates are included in CMS payment reduction programs, with financial penalties for poorer performance

Notes

Author declares no conflicts of interest and that no financial assistance was taken from any source. 


\section{References:}

1. Cohen SH, Gerding DN, Johnson S, et al. Clinical Practice Guidelines for Clostridium difficile Infection in Adults: 2010 update by the Society for Healthcare Epidemiology of America (SHEA) and the Infectious Diseases Society of America (IDSA). Infection Control and Epidemiology 2010; 31(5):431-455.

2. Lessa FC, Mu Y, Bamberg WM, et al. Burden of Clostridium difficile Infection in the United States. New England Journal of Medicine 2015;372:825-34.

3. Dubberke ER, Carling P, Carrico R, et al. Strategies to Prevent Clostridium difficile Infections in Acute Care Hospitals: 2014 Update. Infection Control and Hospital Epidemiology 2014;35(6):628-645.

4. National Healthcare Safety Network (NHSN) Patient Safety Component Manual: Multidrug-Resistant Organism \& Clostridium difficile Infection (MDRO/CDI) Module. January 2016; 12:1-42. http://www.cdc.gov/nhsn/pdfs/pscmanual/pcsmanual_current.pdf

5. Cepheid (May 2010). CXCDIFF/EPI-10 300-9680 Rev. B. Sunnyvale, CA: Cepheid.

6. Crobach MJT, Dekkers OM, Wilcox MH, Kuijper EJ. European Society of Clinical Microbiology and Infectious Diseases (ESCMID): Data review and recommendations for diagnosing Clostridium difficileinfection (CDI). Clinical Microbiology and Infection 2009;15(12):1053-1066.

7. Dubberke ER, Gerding DN. Rationale for Hand Hygiene Recommendations after Caring for a Patient with Clostridium difficile Infection. A Compendium of Strategies to Prevent Healthcare-Associated Infections in Acute Care Hospitals. Fall 2011 Update.

8. Brown KA, Khanafer N, Daneman N, Fisman DN. Meta-analysis of antibiotics and the risk of communityassociated Clostridium difficile infection. Antimicrobial Agents and Chemotherapy 2013;57(5):2326-2332.

9. Slimings C, Riley TV. Antibiotics and hospital-acquired Clostridium difficile infection: update of systematic review and meta-analysis. Journal of Antimicrobial Chemotherapy 2014;69:881-891.

10. O'Horo JC, Jindai K, Kunzer B, Safdar N. Treatment of recurrent Clostridium difficile infection: a systematic review. Infection 2014;42(1):43-59.

11. DHHS Centers for Medicare \& Medicaid Services, 42 CFR §§ 405-412-413 (2016). 